\title{
High Energy Solar Particle Events foRecastlng and Analysis: The HESPERIA Project
}

\author{
O.E. Malandraki* \\ IAASARS, National Observatory of Athens, Athens, Greece \\ E-mail: omalandeastro.noa.gr
}

K.-L. Klein

Observatoire de Paris, Meudon, France

E-mail: ludwig.klein@obspm.fr

\section{R. Vainio}

University of Turku, Finland

E-mail: rami.vainiodutu.fi

\section{N. Agueda}

University of Barcelona, Spain

E-mail: nagueda@am.ub.es

\section{Núñez}

University of Malaga, Spain

E-mail: mnunez@lcc.uma.es

\section{B. Heber}

Christian-Albrechts-Universitaet zu Kiel, Germany

E-mail: heber@physik. uni-kiel.de

\section{R. Buetikofer}

University of Bern, Switzerland

E-mail: rolf.buetikoferespace.unibe.ch

\section{Sarlanis}

ISNet Co, Athens, Greece

E-mail: csarleisnet.gr

\section{N. Crosby}

Belgian Institute for Space Aeronomy, Brussels, Belgium

E-mail: norma.crosby@aeronomie.be

\section{G. Share}

IAASARS, National Observatory of Athens, Athens, Greece

E-mail: gerald.share.ctr@nrl.navy.mil

\section{A. J. Tylka}

United States of America

E-mail: allantylka@yahoo.com 


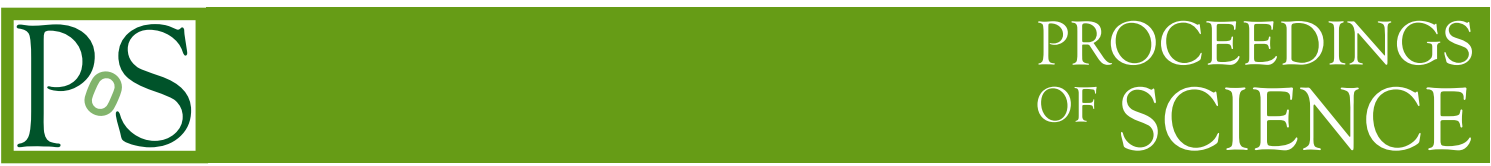

\section{R. Murphy}

Naval Research Laboratory, Washington DC, USA

E-mail: ronald.murphy@nrl.navy.mil

\section{G. Bazilevskaya}

Lebedev Physical Institute of Russian Academy of Sciences, Moscow, Russia

E-mail: gbaz@rambler.ru

\section{Bindi}

University of Hawai at Manoa, Honolulu, USA

E-mail: bindi@hawaii.edu

\section{on behalf of the HESPERIA consortium ${ }^{\dagger}$}

The HORIZON 2020 project 'HESPERIA' is producing two novel solar energetic particle (SEP) operational forecasting tools based upon proven concepts (UMASEP, REleASE). At the same time it will advance our understanding of the physical mechanisms that result into high-energy SEP events through the systematic exploitation of the high-energy $\gamma$-ray observations of the Fermi mission and other novel published datasets (PAMELA; AMS), together with in situ SEP measurements near 1 AU. Furthermore, HESPERIA will explore the possibility to incorporate the derived results into future innovative space weather services. Publicly available software to invert neutron monitor observations of relativistic SEPs to infer the release timescales of relativistic SEPs at the Sun and the characteristics of their transport in the interplanetary space will be provided for the first time. In order to achieve these goals HESPERIA will exploit already available large datasets stored into databases such as the Neutron Monitor DataDase (NMDB) and SEPServer that have been developed under FP7 projects from 2008 to 2013. The structure of the HESPERIA project, its main objectives, as well as the added value to SEP research will be presented and discussed.

The 34th International Cosmic Ray Conference,

30 July-6 August, 2015

The Hague, The Netherlands

${ }^{*}$ Speaker.

${ }^{\dagger}$ www.hesperia-space.eu/index.php/partners 


\section{Introduction}

High-energy particles emitted from the Sun are of great interest both for astrophysics and for space weather applications. From the astrophysics viewpoint the Sun is the unique object where in situ measurements of the particles and remote sensing observations of their radiation can be combined. Both can be carried out with a time resolution that is relevant to the fundamental processes of particle acceleration and transport. From the space weather perspective high-energy particles from the Sun have well-known effects in the Earth's space environment. The impact on the terrestrial atmosphere causes excess ionisation and thereby major disturbances of high-frequency radio communications in polar regions, with increasing concern to civil aviation because of the enhanced traffic on transpolar routes. The impact of energetic particles or the secondaries they produce in the Earth's atmosphere on space borne or air borne electronics constitutes a risk of malfunctioning, and is a threat for spacecraft launch and manoeuvring. Engineering measures have been fruitful in mitigating space weather hazards, but large and extreme events need prediction. These events may also be an excessive radiation threat for human beings aboard transpolar aircraft, and are so for sure for manned space mission crews [1].

HESPERIA (High Energy Solar Particle Events foRecasIng and Analysis) is a two year project that started on 1 May 2015 and is funded through the HORIZON 2020 EU Framework program for research and innovation PROTEC-1-2014: 'Space Weather'. The project combines data and knowledge from 9 European partners and several collaborating parties worldwide. HESPERIA is coordinated by the National Observatory of Athens (NOA) in Greece with Dr. Olga Malandraki as the Project Coordinator. Space weather research at NOA from the solar energetic particle (SEP) perspective within the framework of the two successful FP7 EU projects SEPServer ${ }^{1}$ and COME$\mathrm{SEP}^{2}$ is reviewed in [2].

HESPERIA focuses at high-energy SEP events. The aim of HESPERIA is to unfold both the physical processes that produce these events but at the same time to build novel forecasting operational services. The scientific community has addressed the problem of the origin, acceleration and propagation of high-energy SEPs in a fragmented approach, so far. Within this project, the HESPERIA consortium will utilize novel datasets, employing detailed modelling and data driven analysis to identify the underlying physical processes. Furthermore, proven operational forecasting tools with a successful history will be further improved either by adapting new datasets as inputs to the algorithms or expanding the codes capabilities. In the following, we describe the structure of the HESPERIA project, its main goals and the new insight expected into the physics of SEP events.

\section{Forecasting of solar radiation storms}

Operational real-time SEP forecasts are currently supported by empirical models, which rely on observations of associated solar phenomena, including electromagnetic signatures of SEP acceleration/escape near the Sun, and observations at the near-Earth environment (L1 or $1 \mathrm{AU}$ ) of energetic particles (relativistic electrons or protons). In [3], an empirical electron-based SEP prediction model exploited the shorter transit time of electrons relative to ions. This approach, developed

\footnotetext{
${ }^{1}$ http: //server.sepserver.eu/

${ }^{2}$ http: //www. comesep.eu/
} 

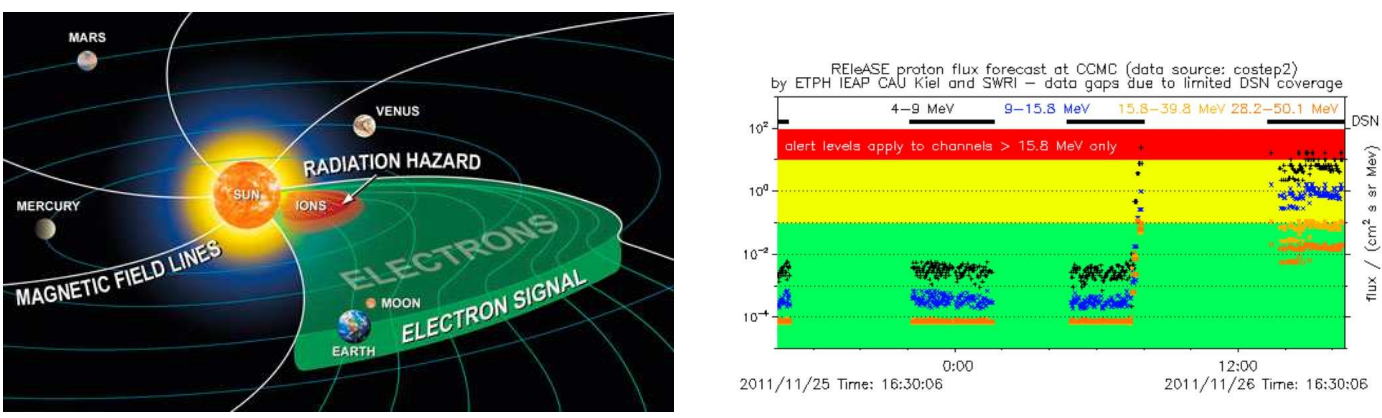

Figure 1: Left: Cartoon of SEPs in the interplanetary space, adapted from [4]; Right: RELeASE forecasting tool graphical output.

into the Relativistic Electron Alert system for Exploration (RELeASE) system, is based on the instrument COSTEP (SOHO), which provides data on relativistic electrons and $<50 \mathrm{MeV}$ protons (see Fig. 1 left). The RELeASE algorithm [3,4] provides a high performance for predicting wellconnected 30-50 MeV SEPs with Probability of Detection (POD) of 85\% and False Alarm Rate (FAR) of 35\%. Figure 1, right, shows the RELeASE graphical output for nowcasting/forecasting 30-50 MeV events ${ }^{3}$.

Another forecasting system is Universidad de Malaga SEP (UMASEP) [5]. It predicts solar proton fluxes $>10 \mathrm{MeV}$ and $>100 \mathrm{MeV}$, by means of analyzing flare and near-Earth space environment data (soft X-ray, differential and integral proton fluxes), Fig. 2. UMASEP has a forecasting model that identifies precursors of well-connected events by empirically estimating the magnetic connectivity from the associated coronal mass ejection (CME)/flare process zone to the near-Earth environment. By taking into account historical and real-time events from 1994 to 2014, the POD for predicting $>10 \mathrm{MeV}$ of the tool is $86.6 \%$ and the FAR $23.1 \%$.

In HESPERIA, we will construct several SEP forecasting tools. One of them is a real-time tool for predicting 30-50 MeV SEP events by using the RELeASE scheme [4]. The new aspect here is that the continuous set of near relativistic electron measurements by ACE/EPAM will be utilized for the first time to infer proton time profiles at $30-50 \mathrm{MeV}$. Furthermore, the implementation and evaluation of a real-time predictor of proton events with energies $>500 \mathrm{MeV}$ by using the UMASEP scheme will be carried out. This tool will correlate solar data and near-Earth radiation data. For the case of real-time solar data SXR, HXR, and radio data be will explored. For the case of real-time near-Earth radiation data, the use of neutron monitor and GOES/HEPAD data will be explored. The developed tool will make real-time predictions of the intensity of the first hours of $>500 \mathrm{MeV}$ proton events. On the other hand, an innovative feasibility study on the usage of microwave data to SEP forecasting will be provided. The aim is to make the forecasting scheme less vulnerable to space weather hazards by including ground-based observations. All these separate tools for large and high-energy SEP events developed in the HESPERIA project will then be integrated in an harmonic package. A web-based interface will be available to access every particular tool's graphical user interface.

\footnotetext{
${ }^{3}$ http://ccmc.gsfc.nasa.gov/models/modelinfo.php?model=REleASE
} 

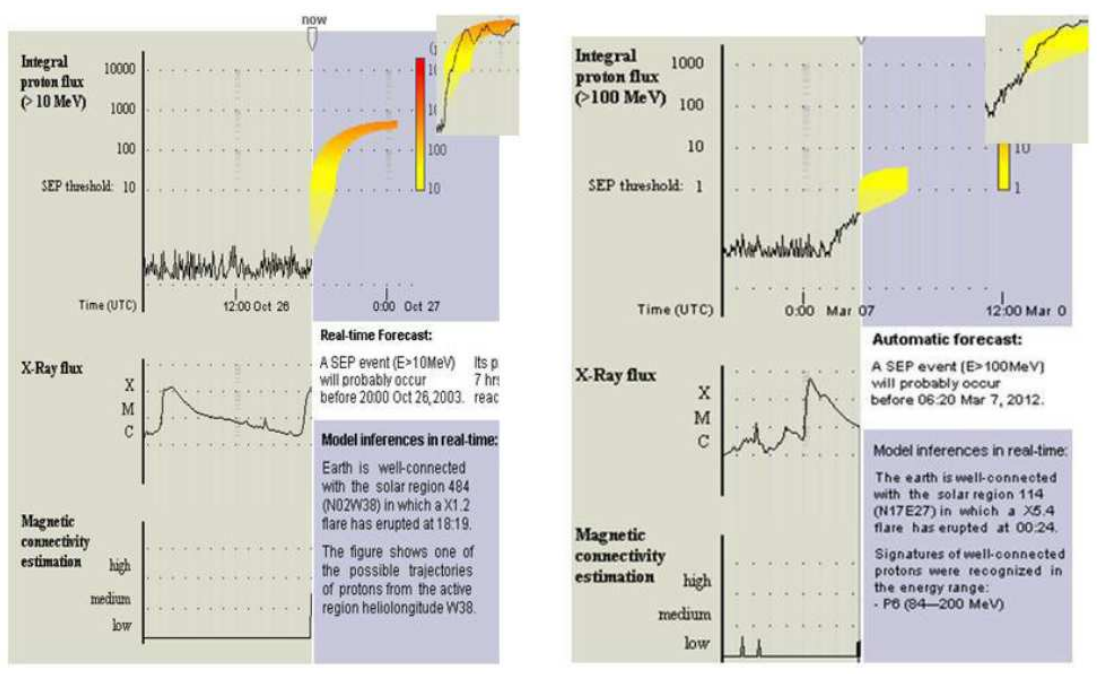

Figure 2: UMASEP forecasting tool graphical interface.

\section{Gamma-ray, radio and SEP observations of relativistic events}

The detailed comparison between electromagnetic (EM) observations and SEP signatures is still not common. It has often been limited in scope by an observational bias: radiative diagnostics of energetic particles in the solar atmosphere refer mostly to electrons (hard X-ray and radio emission), whereas the most relevant space weather hazard of SEPs is due to protons or heavy ions. There is evidence for the contribution of different acceleration regions to SEP events, including, on the one hand, solar flares (SFs) and the related small-scale processes and on the other hand shock waves driven by fast CMEs. From one side, the evidence comes from studies of statistical correlations between parameters of the SEPs and parameters of the eruptive solar activity, including CME speeds [6] and EM signatures of particles in the solar atmosphere [7, 8, 9, 10]. A different approach is based on data driven analyses of individual events, where the early onset of SEP events is compared with the timing of radiative signatures of particles - mostly electrons - in the solar atmosphere and of the associated CME. Finally, modelling of the interplanetary transport, by which the SEP release time profile near the Sun is derived from the intensity and anisotropy time profiles measured in space, and comparison of the derived injection function with radiative signatures of accelerated particles at the Sun is necessary. Part of the scientific results have found their way into forecasting procedures. Observational ingredients are real-time observations of electrons, protons, or electromagnetic emissions - mostly soft X-rays [11,12] - as indicators of energy release at the Sun. Sophisticated schemes employ a combination of these signatures. A crucial lack of existing forecasting schemes is the fact that all radiative signatures employed so far were related to electrons, never to high-energy protons. This has changed with the advent of the Fermi mission providing novel $\gamma$-ray observations (Fermi/LAT) at unprecedented high energies (>100 MeV) [13].

The HESPERIA project will carry out data-driven analyses of Fermi $\gamma$-ray and complementary electromagnetic emissions (hard X-rays, radio waves) in conjunction with SEP observations. This will be the first space weather related analysis that employs high-energy proton signatures from the low solar atmosphere to $1 \mathrm{AU}$. We furthermore want to investigate the potential of this 
radiative signature of protons for SEP forecasting. A multi-frequency analysis of the gamma ray events will also be conducted with the aim to identify possible proxies that can be used in SEP forecasting. Proxies will be necessary, because one cannot count on the continued presence of highly sophisticated instruments like the Fermi mission for space weather purposes. Finally, selected EM signatures will be used as input parameters of the shock-acceleration modelling of these high-energy protons to be carried out within HESPERIA (see next section).

\section{Shock-accelerated SEP modelling of selected Fermi/LAT events}

The novel $\gamma$-ray observations by Fermi/LAT, taken in a systematic way at unprecedentedly high energies, have presented a puzzle to the SEP research community. The Fermi/LAT observations indicate that particles are precipitating to the solar atmosphere for up to a day after the impulsive phase of the flare, which is very difficult to reconcile with a model of impulsive acceleration followed by trapping in the coronal magnetic field. On the other hand, CME-driven shock waves can emit SEPs accelerated up to hundreds of MeVs (in the extreme cases) for hours or even a day after the onset of the associated solar eruption. Therefore, as an alternative view on the genesis of the long-duration $\gamma$-ray events, coronal shock acceleration needs to be considered.

One of the challenges of the shock-acceleration hypothesis is that the SEP events observed in connection with the Fermi/LAT $\gamma$-ray events are not always very large, nor do they extend to very high energies when observed at $1 \mathrm{AU}$. Therefore, one of the key aspects to understand about these events is the spatial distribution of the accelerated particles at the CME-driven shock wave as well as the relation between the interplanetary and interacting protons. Several things contribute to this relation, e.g., the fact that in situ observations are local, while high-energy $\gamma$-ray observations are averaged over all field lines connected to the emission region. Furthermore, the particle spectrum at the shock has to be transported to upstream and downstream to arrive at the fluxes of protons at the Sun and at $1 \mathrm{AU}$ [14]. The particles can also modify their own transport conditions upstream of the shock via streaming instabilities of Alfvén waves, so the fluxes observed at $1 \mathrm{AU}$ can be partially decoupled from the fluxes at the shock. A recently discovered connection between the maximum energy in the escaping proton spectrum and seed particle flux to the shock will also be taken into account [15].

The modelling to be carried out in HESPERIA has the capability to provide a decisive step in understating the origin and connection of near-relativistic solar proton events and the related $\gamma$-ray events that will open new possibilities in remote diagnostics and prediction of SEP events.

\section{Inversion methods for source and transport parameters of relativistic SEPs}

Our knowledge of the processes that release relativistic SEPs into interplanetary space has been so far acquired focusing on three isolated parts: the SEP electromagnetic emissions at the Sun, their detection in the heliosphere by spacecraft instruments, and the ground level enhancements (GLEs) produced by secondaries and observed by the worldwide network of neutron monitors (NMs). Only relatively limited joint efforts between the solar, the heliospheric and the NM communities have been promoted so far. 
The analysis of GLEs based on NM observations has a long tradition. The characteristics of primary particles at the top of the Earth, i.e. energy spectra and directional distributions, are reconstructed from ground-based NM observations taking into account the transport of the cosmic ray particles in the Earth's magnetosphere and atmosphere, and the response of NMs to the secondary atmospheric cosmic ray particles on ground [16, 17]. Spacecraft observations above the Earth's atmosphere can provide valuable information to GLE analysis about the SEP spectrum and the directional distribution of relativistic SEPs before they strike the Earth, as well as information about the direction of the interplanetary magnetic field vector. Moreover, over the last years, members of the HESPERIA consortium developed an inversion methodology for the heliospheric community to infer the release time history of SEPs at the Sun from spacecraft observations [18, 19]. This was developed during a previous FP7 project, SEPServer, and released as a free open software package. The method fits spacecraft observations using results of an interplanetary transport model [20] by solving a linear non-negative least squares optimization problem. In addition to the interplanetary transport conditions, the detector response is taken into account.

Within HESPERIA, we will develop an inversion methodology for NM observations that gives information about the release processes of relativistic SEPs at the Sun and their transport in the interplanetary space. For the first time, publicly available software to invert neutron monitor observations of relativistic SEPs will be provided. Space-borne measurements at lower energies will be used to validate the software for several GLE events.

\section{Assessment of the radiation impact of SEP events}

With the growth of the solar and space physics sector, new knowledge on solar phenomena SFs and CMEs - and sophisticated instrumentation has been made available (X-ray instruments, coronagraphs). Therefore, today, we are in place to also include further indices (e.g., SF rate, CME rate, SF and CME characteristics) into the tracking of the solar cycle dynamics. Large SEP events are usually correlated with CMEs and are referred to as 'gradual' [21]. Those exhibit distinct characteristics and are associated to shock waves, driven out from the Sun by CMEs sampling ion abundances. Gradual events produce by far the highest SEP intensities near Earth [22] and thus are of space weather relevance. Although the number of large SEP events is similar to that in Cycle 23, the Cycle 24 events are generally of lower intensity. Observations show that the overall CME rate is not lower during Cycle 24, as compared to the one achieved in solar cycle 23. Measurements demonstrate that the fraction of full halo CMEs among the SEP events has been reported being much larger during Cycle 24 [23]. However, the CME speeds are similar during both cycles. Consequences for the maximum energy attainable from acceleration by transient shocks, including those driven by CMEs, arise.

Within HESPERIA we plan to perform an in depth statistical analysis in order to establish relations among the parent solar events and the derived SEPs including a thorough investigation on compositional signatures. Furthermore, modelling on long time-scale will be employed to demonstrate how the maximum energy attainable from acceleration by transient shocks under weak magnetic field conditions is affected. 


\section{Summary}

In this paper, an overview of the Horizon 2020 'HESPERIA' project has been presented. HESPERIA intends to provide new insight into the physics of SEP events and to develop improved and novel tools for SEP analysis and forecasting:

- The project will for the first time conduct systematic research on protons from the Sun to $1 \mathrm{AU}$, through a systematic exploitation of the new high-energy $\gamma$-ray observations of the Fermi mission together with in situ SEP measurements near 1 AU.

- The project will address the chain of processes from particle acceleration in the corona, particle transport in the magnetically complex corona and interplanetary space to the detection near 1 AU through multi-frequency observations and simulations.

- The project will compare, develop and validate SEP forecasting tools through the new input from the above research and by investigating proxies that can be provided by simple patrol instruments, both in space and on ground. This will lead to a better understanding of forecasting schemes and to policy recommendations for future space weather services.

- The project will provide for the first time publicly available software to invert neutron monitor observations of relativistic SEPs to infer the release timescales of relativistic SEPs at the Sun and the characteristics of their transport in the interplanetary space.

In addition the consortium will provide educational and outreach material on solar eruptions and the space environment on its website: www. hesperia-space.eu.

\section{Acknowledgments}

This project has received funding from the European Union's Horizon 2020 research and innovation programme under grant agreement No 637324.

\section{References}

[1] N. B. Crosby, Space weather: science and effects, in IAU Symposium (N. Gopalswamy and D. F. Webb, eds.), vol. 257 of IAU Symposium, pp. 47-56, Mar., 2009.

[2] O. E. Malandraki, Space Weather Research in Greece: The Solar Energetic Particle Perspective, Sun and Geosphere 10 (Mar., 2015) 21-30.

[3] A. Posner, Up to 1-hour forecasting of radiation hazards from solar energetic ion events with relativistic electrons, Space Weather 5 (May, 2007) 5001.

[4] A. Posner, S. Guetersloh, B. Heber, and O. Rother, A New Trend in Forecasting Solar Radiation Hazards, Space Weather 7 (May, 2009) 5001.

[5] M. Núñez, Predicting solar energetic proton events (E > 10 MeV), Space Weather 9 (July, 2011) 7003.

[6] S. W. Kahler, The correlation between solar energetic particle peak intensities and speeds of coronal mass ejections: Effects of ambient particle intensities and energy spectra, J. Geophys. Res. 106 (Oct., 2001) 20947-20956. 
[7] H. A. Garcia, Forecasting methods for occurrence and magnitude of proton storms with solar soft $X$ rays, Space Weather 2 (Feb., 2004) 2002.

[8] I. M. Chertok, V. V. Grechnev, and N. S. Meshalkina, On the correlation between spectra of solar microwave bursts and proton fluxes near the Earth, Astronomy Reports 53 (Nov., 2009) 1059-1069.

[9] J. A. Grayson, S. Krucker, and R. P. Lin, A Statistical Study of Spectral Hardening in Solar Flares and Related Solar Energetic Particle Events, Astrophys. J. 707 (Dec., 2009) 1588-1594.

[10] I. M. Chertok, On the correlation between the solar gamma-ray line emission, radio bursts and proton fluxes in the interplanetary space, Astronomische Nachrichten 311 (Oct., 1990) 379-381.

[11] S. W. Kahler, E. W. Cliver, and A. G. Ling, Validating the proton prediction system (PPS), Journal of Atmospheric and Solar-Terrestrial Physics 69 (Feb., 2007) 43-49.

[12] C. C. Balch, Updated verification of the Space Weather Prediction Center's solar energetic particle prediction model, Space Weather 6 (Jan., 2008) 1001.

[13] M. Ackermann et al., High-energy Gamma-Ray Emission from Solar Flares: Summary of Fermi Large Area Telescope Detections and Analysis of Two M-class Flares, Astrophys. J. 787 (May, 2014) 15.

[14] L. Kocharov, T. Laitinen, R. Vainio, A. Afanasiev, K. Mursula, and J. M. Ryan, Solar Interacting Protons Versus Interplanetary Protons in the Core Plus Halo Model of Diffusive Shock Acceleration and Stochastic Re-acceleration, Astrophys. J. 806 (June, 2015) 80.

[15] R. Vainio, A. Pönni, M. Battarbee, H. E. J. Koskinen, A. Afanasiev, and T. Laitinen, A semi-analytical foreshock model for energetic storm particle events inside 1 AU, Journal of Space Weather and Space Climate 4 (Feb., 2014) A8.

[16] R. Bütikofer, E. O. Flückiger, L. Desorgher, M. R. Moser, and B. Pirard, The solar cosmic ray ground-level enhancements on 20 January 2005 and 13 December 2006, Advances in Space Research 43 (2009) 499-503.

[17] R. Bütikofer and E. O. Flückiger, Differences in published characteristics of GLE60 and their consequences on computed radiation dose rates along selected fight paths, Journal of Physics Conference Series 409 (2013), no. 1012166.

[18] N. Agueda, R. Vainio, D. Lario, and B. Sanahuja, Injection and interplanetary transport of near-relativistic electrons: Modeling the impulsive event on 2000 May 1, Astrophys. J. 675 (2008) 1601-1613.

[19] N. Agueda, K.-L. Klein, N. Vilmer, R. Rodríguez-Gasén, O. E. Malandraki, A. Papaioannou, M. Subirà, B. Sanahuja, E. Valtonen, W. Dröge, A. Nindos, B. Heber, S. Braune, I. G. Usoskin, D. Heynderickx, E. Talew, and R. Vainio, Release timescales of solar energetic particles in the low corona, Astron. Astrophys. $\mathbf{5 7 0}$ (2014) A5.

[20] N. Agueda, R. Vainio, and B. Sanahuja, A Database of $>20$ keV Electron Green's Functions of Interplanetary Transport at 1 AU, Astrophys. J. Suppl. 202 (2012) 18.

[21] D. V. Reames, Particle acceleration at the Sun and in the heliosphere, Space Sci. Rev. 90 (Oct., 1999) 413-491.

[22] D. V. Reames, The Two Sources of Solar Energetic Particles, Space Sci. Rev. 175 (June, 2013) 53-92.

[23] N. Gopalswamy, H. Xie, S. Akiyama, P. Mäkelä, S. Yashiro, and G. Michalek, The Peculiar Behavior of Halo Coronal Mass Ejections in Solar Cycle 24, Astrophys. J. Lett. 804 (May, 2015) L23. 